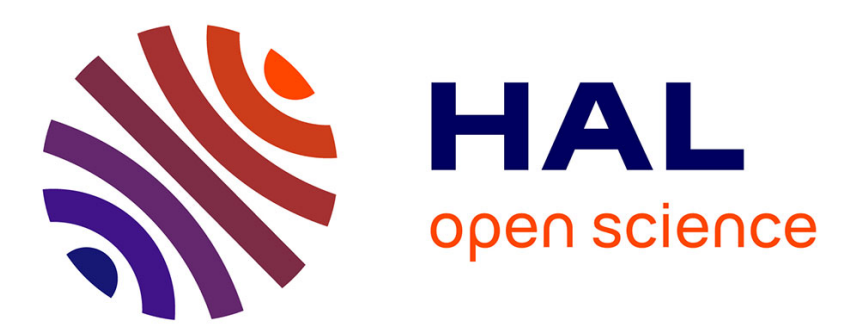

\title{
Characterization and mapping of sleep-waking specific neurons in the basal forebrain and preoptic hypothalamus in mice.
}

Kasumi Takahashi, Jian-Sheng Lin, Kazuya Sakai

\section{- To cite this version:}

Kasumi Takahashi, Jian-Sheng Lin, Kazuya Sakai. Characterization and mapping of sleep-waking specific neurons in the basal forebrain and preoptic hypothalamus in mice.. Neuroscience, 2009, 161 (1), pp.269-92. 10.1016/j.neuroscience.2009.02.075 . inserm-00383292

\section{HAL Id: inserm-00383292 https://www.hal.inserm.fr/inserm-00383292}

Submitted on 18 May 2009

HAL is a multi-disciplinary open access archive for the deposit and dissemination of scientific research documents, whether they are published or not. The documents may come from teaching and research institutions in France or abroad, or from public or private research centers.
L'archive ouverte pluridisciplinaire HAL, est destinée au dépôt et à la diffusion de documents scientifiques de niveau recherche, publiés ou non, émanant des établissements d'enseignement et de recherche français ou étrangers, des laboratoires publics ou privés. 


\title{
CHARACTERIZATION AND MAPPING OF SLEEP-WAKING SPECIFIC NEURONS IN
} THE BASAL FOREBRAIN AND PREOPTIC HYPOTHALAMUS IN MICE

\author{
Kazumi TAKAHASHI ${ }^{\dagger *}$, Jian-Sheng LIN $^{\dagger}$ and Kazuya SAKAI ${ }^{\dagger}$ \\ ${ }^{\dagger}$ INSERM U628, Lyon, F-69373 and Claude Bernard University Lyon 1, 8 Avenue Rockefeller, Lyon Cedex 08, \\ F-69373, France \\ *Department of Physiology, Fukushima Medical University, 1 Hikarigaoka, Fukushima 960-1295, Japan
}

Running title: Sleep-waking discharge of preoptic/basal forebrain neurons

Total number of Figures: 19

Total number of Tables: 1

Number of pages: 35

Corresponding author:

K. Sakai

INSERM U628, Université Claude Bernard, Lyon 1

8 Avenue Rockefeller, 69373 Lyon Cedex 08, France

Tel: +(33) 4787771 22; Fax: +(33) 478777151

E-mail: sakai@univ-lyon1.fr

Key words: mouse, single unit recording, sleep-waking states, preoptic area, basal forebrain

\section{Acknowledgements:}

This study was supported by INSERM U628 and Claude Bernard University. K. Takahashi was supported by grants from the Fyssen Foundation. 


\section{Abbreviations:}

AHA, anterior hypothalamic area; AW, active waking; BFB, basal forebrain; ChAT, choline acetyltransferase;

$\mathrm{CV}$, coefficient of variation; D, drowsy state; EEG electroencephalogram, EMG, electromyogram; GABA, gamma-aminobutyric acid; Glu, glutamate; HA, histamine, HDB, nucleus of the diagonal band of Broca; IMEANFR, instantaneous mean frequency; LH, lateral hypothalamic area; LTS, low threshold spike; MCPO, magnocellular preoptic nucleus; MPO, medial preoptic area; NA, noradrenaline; Orx/Hcrt, orexin/hypocretin; POA, preoptic area; PH, posterior hypothalamus; QW, quiet waking; SI, substantia innominata; SWS, slowwave sleep; TI-S and TI-R, slowly and rapidly discharging tonic type I neurons, respectively; TI-Ss and TI-Rs, TI-S and TI-R neurons discharging specifically in association with cortical activation during both waking and PS, respectively; VLPO, ventrolateral preoptic nucleus; vlPOA, ventrolateral preoptic area; W, wakefulness 


\begin{abstract}
We recorded 872 single units across the complete sleep-waking cycle in the mouse preoptic area (POA) and basal forebrain (BFB), which are deeply involved in the regulation of sleep and wakefulness (W). Of these, 552 were sleep-active, 96 were waking-active, 106 were active during both waking and paradoxical sleep (PS), and the remaining 118 were state-indifferent. Among the 872, we distinguished slow-wave sleep (SWS)-specific, SWS/PS-specific, PS-specific, W-specific, and W/PS-specific neurons, the last group being further divided into TI-Ss and TI-Rs. Both the SWS/PS-specific and PS-specific neurons were distributed throughout a wide region of the POA and BFB, whereas the SWS-specific neurons were mainly located in the middle and ventral half of the POA and adjacent BFB, as were the W-specific and W/PS-specific neurons. At the transition from waking to sleep, the majority of SWS-specific and all SWS/PS-specific neurons fired after the onset of cortical synchronization (deactivation), whereas all W-specific and W/PS-specific neurons showed a significant decrease in firing rate $>0.5 \mathrm{~s}$ before the onset. At the transition from SWS to $\mathrm{W}$, the sleep-specific neurons showed a significant decrease in firing rate $0.1 \mathrm{~s}$ before the onset of cortical activation, while the W-specific and W/PSspecific neurons fired $>0.5 \mathrm{~s}$ before the onset. TI-Ss neurons were characterized by a triphasic broad action potential, slow single isolated firing, and an antidromic response to cortical stimulation, whereas TI-Rs neurons were characterized by a narrow action potential and high frequency burst discharge in association with theta waves in PS. These data suggest that the forebrain sleep/waking switch is regulated by opposing activities of sleep-promoting (SWS-specific and SWS/PS-specific) and waking-promoting (W-specific and W/PS-specific) neurons, that the initiation of sleep is caused by decreased activity of the waking-promoting neurons (disfacilitation), and that the W/PS-specific neurons are deeply involved in the processes of cortical activation/deactivation.
\end{abstract}

\title{
(299 words)
}




\section{Introduction}

The preoptic area (POA) of the anterior hypothalamus and the adjacent basal forebrain (BFB) play an important role in the regulation of homeostatic and cognitive processes, including blood pressure, thermoregulation, sexual behavior, attention, learning, and cortical activity (Boulant and Dean, 1986; Everitt, 1990; Everitt and Robbins, 1997; Jones, 2004). Both regions are deeply involved in the regulation of sleepwaking states. On the basis of neuropathological studies in humans, von Economo (von Economo, 1930) proposed, early in the $20^{\text {th }}$ century, the concept of opposing hypothalamic sleep-promoting and wakingpromoting systems, because patients suffering from severe insomnia were found on postmortem to have inflammatory lesions in the POA, whereas those suffering from severe hypersomnia before death had lesions in the vicinity of the posterior hypothalamus (PH). This concept was supported by early experimental studies (Moruzzi, 1972; Nauta, 1946) and confirmed by recent studies in animals: destruction or inactivation of posterior hypothalamic neurons results in somnolence and hypersomnia (Lin et a1., 1989; Sakai et al., 1990), whereas similar treatment of POA/BFB neurons leads to insomnia (Lin et al., 1989; McGinty and Sterman, 1968; Sakai et al., 1990; Sallanon et al., 1989). The existence of sleep-promoting mechanisms in the POA and wakingpromoting mechanisms in the $\mathrm{PH}$ has been demonstrated by a variety of experimental studies, including lesion, stimulation, microinjection of neurochemical agents, single unit recording, c-fos expression, and genetic approaches (Huang et al., 2007; Jones, 2005; Lin, 2000; McGinty and Szymusiak, 2001; Sakai et al., 1990; Sakurai, 2002; Saper et al., 2001).

Recently, based on the sleep-related expression of the immediate early gene c-fos, a marker of neuronal activation, a restricted collection of neurons has been described in the ventrolateral region of the POA, referred to as the "ventrolateral preoptic nucleus (VLPO)" (see, however, Fig. 1), and these were proposed to be the principal sleep-promoting cell group within the POA and BFB (Sherin et al., 1996). More recent c-fos studies found other sleep-promoting cell groups, as well as waking-promoting ones, in the POA and BFB (Gong et al., 2000; Gvilia et al., 2006; Modirrousta et a1., 2004). However, c-fos expression studies are not suitable for revealing neuronal discharge activity during the sleep-waking states. The sleep/waking-related neuronal activity of POA/BFB neurons can only be determined by recording their unit activity during the sleep-waking cycle. Single unit recording studies in naturally waking-sleeping cats and rats have reported units that discharge at the highest rate during either cortical slow wave activity, observed during slow-wave sleep (SWS), or cortical activation (desynchronization), occurring during wakefulness (W) and/or paradoxical sleep (PS) (Alam et al., 1996; Détari et al., 1984; Koyama and Hayaishi, 1994; Lee et al., 2004; Osaka and Matsumura, 1994; Szymusiak 
and McGinty, 1986). However, the precise anatomical distribution of these neurons in the POA and BFB and their unit activity profiles during state transitions are still uncertain, but this knowledge is a prerequisite for an understanding of the generation and/or maintenance of behavioral states. In addition, no information is available about the activity of sleep/waking-related neurons in the POA and BFB of the mouse, despite its increasing use in experimental models. In our recent studies on the mouse PH (Takahashi et al., 2006, 2008), we reported the activity profiles of waking-specific histamine (HA) neurons and waking-active orexin (also called hypocretin, Hcrt) (Orx/Hcrt) and non-Orx/Hcrt neurons during state transitions. One of the main goals of the present study was to compare the activity profiles of sleep-active neurons in the POA/BFB and those of the waking-specific and waking-active neurons in the $\mathrm{PH}$ in order to elucidate the hypothalamic switch mechanism underlying the transition of behavioral states, i.e., from waking to sleeping and from sleeping to waking. In the present study, therefore, we used extracellular single unit recordings in unanesthetized, head-restrained mice to record a large number of single units in the POA and the adjacent BFB and determined their activity profiles during all sleepwaking states and at state transitions in an attempt to i) identify sleep-promoting and waking-promoting neurons, ii) determine their anatomical distribution, and iii) elucidate the hypothalamic mechanism of sleep and the waking switch. 


\section{Experimental Procedures}

\section{Animals and Surgery}

All procedures were approved by the University of Lyon 1 Animal Care Committee, the standards of which meet those of the EEC Guidelines (86/609/EEC) and the Policy on Ethics approved by the Society for Neuroscience (1993). All efforts were made to minimize the number of animals used and their suffering.

Twenty-three male adult C57BL/6 mice (Harlan, France; 26-35 g at the time of surgery) were used. The mice were anesthetized using a mixture of $0.8 \mathrm{mg} / \mathrm{ml}$ ketamine and $1 \mathrm{mg} / \mathrm{ml}$ xylazine at an initial dose of 10 $\mathrm{ml} / \mathrm{kg}$, with $3 \mathrm{ml} / \mathrm{kg}$ boosters as needed, given i.p. . The mice were placed in a stereotaxic apparatus (SN-3, Narishige, Tokyo, Japan) with blunt ear bars and implanted with electrodes to record the neocortical electroencephalogram (EEG), neck electromyogram (EMG), and electrocardiogram (EKG), as described previously (Takahashi et al., 2008). Bipolar stimulating electrodes, consisting of two Formvar-coated, stainless steel wires $(120 \mu \mathrm{m}$ in diameter, $0.5 \mathrm{~mm}$ apart, bared $0.25 \mathrm{~mm}$ at the tip) were placed in the medial frontal cortex (AP $2.5 \mathrm{~mm}$ from the bregma, ML $0.5 \mathrm{~mm}$ lateral and $1.2 \mathrm{~mm}$ ventral to the brain surface). One cannula (30 gauge) was fixed on the skull (AP $2.0 \mathrm{~mm}$ from the bregma and ML $0.0 \mathrm{~mm}$ ) and used as a stereotaxic reference during the experiment. In addition, a U-shaped plastic plate (18 mm wide, $16 \mathrm{~mm}$ long, $5 \mathrm{~mm}$ thick) was fixed stereotaxically to the skull using dental acrylic cement so that the cranium could be painlessly returned to the same stereotaxic position using a chronic head holder (SA-8, Narishige). A small hole was drilled in the skull above the basal forebrain and preoptic area and covered with antibiotic cream for the subsequent insertion of microelectrodes. After a recovery period of one week, the animals were habituated progressively to the headrestrained position (7-14 days) by placing them on a cotton sheet inside a plastic box, painlessly restraining the head with a head holder, and preventing large body movements with a cotton-coated plastic covering. The head was covered to reduce visual stimuli and a clean part of the cotton sheet was kept in contact with the mouse by sliding the sheet, if necessary, during the experiment. Under these conditions, the animals were able to move their bodies and limbs relatively freely and to sleep in a sphinx position. After habituation, they could be kept in this position for 3-6 consecutive hours without showing any signs of discomfort and displayed complete sleepwaking cycles, consisting of W, SWS, and PS. If any signs of discomfort were seen, the mouse was freed from the restrained position. Animals were maintained on a $12 \mathrm{~h} \mathrm{light/dark} \mathrm{schedule} \mathrm{with} \mathrm{lights} \mathrm{on} \mathrm{from} 7$ a.m. to 7 p.m., and recordings were usually made between 10 and 12 a.m. and between 2 and 5 p.m., periods in which mice normally sleep 


\section{Extracellular single unit and polygraphic recordings}

Single neuronal activity was recorded extracellularly using a glass pipette microelectrode filled with 0.5 M sodium acetate solution containing 2\% Pontamine Sky Blue as described previously (Takahashi et al. 2006). The microelectrode was attached perpendicular to the electrode holder of a pulse motor microdrive manipulator (MO-81, Narishige). After determination of the AP and ML stereotaxic coordinates using the guide cannula as a reference, the electrode was placed over a target structure. The exposed brain surface was cleaned and $1 \%$ xylocaine was applied to the electrode insertion point. The electrode was then lowered until it touched the brain surface using an electrode carrier (SM-15, Narishige) equipped with a substage (SM-15M, Narishige), permitting a fine movement $(10 \mu \mathrm{m})$ in the DV or AP direction. The DV stereotaxic coordinate at the brain surface was noted. During unit recordings, the electrode was then inserted in the brain using the microdrive manipulator in increments of 1-3 $\mu \mathrm{m}$, initially at $100-200 \mathrm{pps}$, then at 5-15 pps. The exposed brain surface was protected against drying by filling the hole in the skull with physiological saline and applying a gelatin sponge to the brain surface. When single unit activities were recorded in the POA and BFB, the driven distance of the electrode tip from the brain surface (displayed on the remote control unit of the microdrive manipulator) was noted and unit recordings were carried out during at least one complete sleep-waking cycle lasting 5-20 min. The neuronal activity was amplified and filtered (NeuroLog, Digitimer, Hertfordshire, UK) with a cut-off frequency of $100 \mathrm{~Hz}$, then digitized at a sampling rate of $20.8 \mathrm{kHz}$ using a CED 1410 data processor (Cambridge Electronic Design (CED), Cambridge, UK). The polygraphic signals were also digitized at a sampling rate of $508.1 \mathrm{~Hz}$ and stored on a personal computer.

Unit recordings were usually made bilaterally at intervals of $0.3 \mathrm{~mm}$ rostrocaudally and mediolaterally. At the end of each experiment, Pontamine Sky Blue was injected from the recording electrode by passing a negative current (5 $\mu \mathrm{A}$ for 5-6 min) so as to mark one or two recording sites, the location of which was previously determined by the microdrive manipulator, in each electrode track. During a series of laterally aligned unit recordings, the electrode was occasionally removed from the brain without marking any recording sites with dye, placed $0.2-0.3 \mathrm{~mm}$ medial or lateral to the previous electrode track, and lowered by the electrode carrier to the same DV coordinate as that determined in the first recording trial. At the end of the experiment, one or two recording sites were then marked with Pontamine Sky Blue in the last track as described above, allowing the location of the unit recording sites in the previous electrode track(s) to be determined histologically (see below). After each experimental session, the exposed brain surface was cleaned and covered with antibiotic cream after local application of xylocaine. Unit recordings were made during two experimental sessions per day and the 
experiment lasted 5-6 consecutive days. The mean ( \pm SD) number of electrode insertions per mouse was $14.2 \pm$ 9.0, the number of units recorded successfully during the complete wake-sleep state per mouse was $38.3 \pm 32.8$, and the number of units per track was $2.7 \pm 1.3$.

\section{Stimulation experiment}

Stimulation was performed in 4 of the 23 mice using square pulses $(0.1-0.2 \mathrm{~ms})$ delivered at $0.6-0.8 \mathrm{~Hz}$ using a WPI 302 stimulator (World Precision Instruments, Florida, USA) and a stimulus isolation unit (Mode1 305-2, WPI). A fixed latency and the collision of spontaneous and stimulation-evoked action potentials (collision test) were used as the criteria for differentiating between antidromic invasion and synaptic activation.

\section{Histochemistry and determination of unit recording sites}

Under deep anesthesia, the animals were perfused through the ascending aorta with $50 \mathrm{ml}$ of Ringer's solution, followed by $120 \mathrm{ml}$ of fixative consisting of $4 \%$ paraformaldehyde and $0.2 \%$ picric acid in $0.1 \mathrm{M}$ phosphate buffer, pH 7.4 (PB). The brain was removed, postfixed for $24 \mathrm{~h}$ at $4^{\circ} \mathrm{C}$ in the same fixative, then placed in PB containing $30 \%$ sucrose for $48 \mathrm{~h}$ at $4^{\circ} \mathrm{C}$. Twenty micrometer coronal sections were cut serially on a cryostat and collected in strict order in $0.1 \mathrm{M}$ phosphate-buffered saline, $\mathrm{pH} 7.4$, containing $0.3 \%$ Triton X-100 (PBST) in the wells of two 12-well tissue culture plates. They were then rinsed and stored at $4^{\circ} \mathrm{C}$ in PBST.

To verify whether or not unit recordings were made in the cholinergic field of the basal forebrain and adjacent preoptic/anterior hypothalamus, sections were incubated for $4-5$ days at $4{ }^{\circ} \mathrm{C}$ with rabbit anti-choline acetyltransferase (ChAT) antibodies (Millipore, Billerica, MA) diluted 1/5,000 in PBST containing $0.1 \%$ sodium azide, then, after several washes, were incubated overnight at $4{ }^{\circ} \mathrm{C}$ with biotinylated anti-rabbit IgG antibodies (Vector Laboratories, Burlingame, CA) diluted 1/1,000 in PBST. After several rinses, they were incubated for 90 min at room temperature with $\mathrm{ABC}$ (Vector Laboratories, diluted 1/1,000), then processed for visualization using DAB-nickel (Vector Laboratories) as chromogen. The sections were mounted in strict rostrocaudal order on gelatin-coated glass slides, dried, stained with Neutral red, dehydrated, cleared, and finally cover-slipped with Depex for observation under a light microscope.

For the reconstruction of the unit recording sites, camera lucida drawings of the sections were made under a light microscope, then brain structures were delineated and all spots marked by Pontamine Sky Blue outlined (see Fig. 4). After identification of each dye deposit, the location of each unit recording site was determined based on the dye marking sites and the sterotaxically determined recording points noted during the 
experiment. Each unit recording site was then plotted on a standard atlas constructed in our laboratory and the corresponding cell number noted beside it (see Fig. 1).

\section{Data analysis}

Sleep-waking stages. Sleep-waking stages were defined using the EEG and neck EMG signals. W was defined as a low-voltage, desynchronized (activated) EEG or theta waves accompanying sustained EMG activity. The drowsy state (D) was defined as the first 3-s period from the onset of EEG synchronization (deactivation) during the transition from W to SWS. SWS was defined by sustained high-voltage slow waves in the EEG and lowered EMG activity. In the present study, D and SWS corresponded, respectively, to light and deep SWS. PS waS defined by sustained theta waves and decreased delta waves in the EEG and the absence of EMG activity. Analysis of unitary activity was performed using Spike2 software (CED). Mean discharge rates were calculated from a total analysis time of greater than $10-30 \mathrm{~s}$ using $2-10 \mathrm{~s}$ bins for each of the following five states: (1) active or attentive waking (AW), characterized by either overt body and limb movements or elicited by touching the tail with a soft brush or removing the cover placed over the head of the mouse $(2-5-\mathrm{s}$ bin). This state is characterized by a low amplitude (desynchronized) EEG with a sustained EMG activity; (2) quiet waking (QW), characterized by the absence of gross movements (3 - 10-s bin); (3) D (3-s bin); (4) SWS (10-s bin); and (5) PS (10-s bin). The coefficient of variation (CV) for the spike interval (standard deviation/mean discharge interval) was used as a quantitative measure of the regularity of unit discharge.

State transitions. Wake-sleep state transitions were determined as described previously (Takahasi et al., 2006, 2008). For the transition between SWS and W, onset of W was determined by the first sign of EEG desynchronization (activation), while onset of sleep was defined by the first appearance of synchronized (deactivated) EEG (see Fig. 5A and B). During the transitions from SWS to PS and from PS to W, onset and end of PS were defined, respectively, by the appearance of continuous rhythmic theta waves on the cortical EEG and by the interruption of sustained theta waves and the onset of desynchronized EEG (see Fig. 5C).

Statistical analysis. Statistical analysis was carried out using either the Kruskal-Wallis test and post hoc MannWhitney test or a two-tailed t test, a $P$ value of $<0.05$ or $<0.01$ being considered, respectively, as significant or highly significant. 


\section{Results}

Extracellular single unit recordings were made from a total of 872 neurons during the complete sleepwaking cycle including at least one PS episode in the POA and adjacent BFB, including the medial (MPO) and lateral POA (LPO), ventrolateral preoptic nucleus (VLPO), ventrolateral preoptic area (vlPOA), substantia innominata (SI), horizontal limb of the diagonal band of Broca (HDB), magnocellular preoptic nucleus (MCPO), and anterior (AHA) and lateral (LH) hypothalamic areas (Fig. 1). As shown in Table 1, these neurons could be divided into 4 groups on the basis of differences in their firing patterns during the sleep-waking cycle: i) those showing the highest rate of spontaneous discharge during sleep (sleep-active neurons; $63.3 \%$ ); ii) those discharging at the highest rate during $\mathrm{W}$ (W-active neurons; $11.0 \%$ ); iii) those showing an increased discharge rate during W and PS compared to SWS (W/PS-active neurons; $12.2 \%$ ); and iv) those showing state-indifferent unit activity (State-unrelated neurons; $13.5 \%$ ).

Insert Figure 1 and Table 1 about here

\section{Sleep-active neurons}

Sleep-active neurons were divided into the 3 subgroups of SWS-active, SWS/PS-active, and Low rate based on differences in their firing patterns and rates (Table 1).

\section{SWS-active neurons}

Ninety-four of the 552 sleep-active neurons (10.8\%) were characterized by having a higher rate of spontaneous discharge during SWS than during W and PS (Table 1). Of these, 65 displayed no, or virtually no, spontaneous discharge during W and fired selectively during SWS compared to PS, the ratio of the firing rate during SWS to that during PS being > 1.2 (Figs. 2 and 3A): these are hereafter referred to as "SWS-specific" neurons. On the basis of their high $(\geq 9 \mathrm{~Hz} ; \mathbf{n}=25)$ or low $(<9 \mathrm{~Hz} ; \mathrm{n}=40)$ spontaneous firing rates during SWS (Fig. 3A), they could be further subdivided into 2 groups, which will be referred to as "fast" and "slow" SWSspecific neurons. The distribution of the SWS-specific neurons is shown on the right half of each section in Fig. 1 (f-j, black squares), while that of the ChAT-immunoreactive, cholinergic neurons is shown on the left half of each section (Fig. 1, a-e, dots and circles; see also Fig. 4). One of the unit recording sites is shown in Fig. 4b (left arrow). The SWS-specific neurons were located ventrally in the vlPOA and HDB, laterally in the MCPO and SI, and more dorsally in the lateral part of the MPO and AHA, and throughout the LPO and LH. They were thus distributed in both cholinergic and non-cholinergic neuron fields. 
Insert Figures 2 and 3 about here

Spike shape and spontaneous discharge rate and pattern of SWS-specific neurons

All the fast SWS-specific neurons were characterized by having a biphasic and narrow action potential. The mean ( \pm SD) spike duration (positive inflection to first zero crossing (D2); see Fig. 14B and C) was $0.52 \pm$ $0.11 \mathrm{~ms}$. Most (38/40) of the slow SWS-specific neurons showed a biphasic spike, the remaining 2 being triphasic. The overall mean $( \pm \mathrm{SD})$ spike duration for all 40 neurons was $0.55 \pm 0.11 \mathrm{~ms}$, not significantly different from that of the fast SWS-specific neurons $(P>0.05$, two-tailed $t$ test).

The mean spontaneous discharge rate of the SWS-specific neurons during the sleep-waking cycle is shown in Fig. 3A. Most (48/65) SWS-specific neurons were completely silent during both AW and QW and displayed a sharp increase in discharge rate at sleep onset (drowsy state, D). They fired at the highest rate during SWS and showed a significant decrease in firing rate or stopped firing during PS. The difference in firing rate between D and SWS was significant for the slow SWS-specific neurons $(P<0.05$, Mann-Whitney), but not for the fast SWS-specific neurons ( $P>0.05$, Mann-Whitney) (Fig. 3A).

As illustrated in Fig. 2, the fast SWS-specific neurons exhibited high frequency burst discharges (peak instantaneous mean frequency (IMEANFR) $>200 \mathrm{~Hz}$ ) during, and at the onset of, SWS. Although 8 of the 40 slow SWS-specific neurons also fired in high frequency bursts, 15 of the remainder fired mainly as either single isolated spikes and 17 as clusters (peak IMEANFR $<150 \mathrm{~Hz}$ ).

Insert Figures 4, 5 and 6 about here

Activity profiles of SWS-specific neurons during wake-sleep state transitions

During the transition from SWS to W, all SWS-specific neurons ceased firing before the onset of EEG activation (Figs. 2 and 5A-1). The mean $( \pm \mathrm{SD})$ interval between the last spike discharge and the onset of EEG activation was $236.7 \pm 143.1 \mathrm{~ms}$. SWS-specific neurons also ceased firing when waking was elicited during sleep by an arousing sound stimulus (hand clapping) (Fig. 6). The mean ( \pm SD) interval between the last unit discharge and the onset of the stimulus was $-42.6 \pm 154.2 \mathrm{~ms}$ for the 10 fast SWS-specific neurons tested.

During the transition from W to D (Fig. 5B-1), 11 of the 25 fast SWS-specific neurons discharged either prior to $(+)$ or after (-) the onset of EEG synchronization (deactivation), the first sign of EEG sleep (D), with a 
mean ( \pm SD) interval of $-83.8 \pm 104.8 \mathrm{~ms}$. The remaining 14 always fired after the onset of EEG synchronization, with a mean latency of $453.7 \pm 261.2 \mathrm{~ms}$. We further estimated the time course of unit discharges from SWS to W and from W to D with 0.1 -s bins for a sample of the 5 most characteristic fast SWSspecific neurons that fired before the onset of D and displayed multiple sleep-waking cycles, providing many state transitions. As shown in Fig. 7, the SWS-specific neurons showed a significant decrease in discharge rate just before $(0.1 \mathrm{~s})$ the onset of $\mathrm{W}$ (EEG activation) (Fig. 7, upper panel) and began to fire as early as $0.2 \mathrm{~s}$ prior to the onset of D (EEG deactivation) (Fig. 7, lower panel).

We then examined the time course of the unit discharge at the transition from SWS to PS $(n=22)$ with 0.5 -s bins. As shown in Figs. 5C-1, 8A-1, and 8B, the SWS-specific neurons showed a significant decrease in firing rate $1.0 \mathrm{~s}$ after the onset of PS, defined by the appearance of continuous rhythmic theta waves.

Insert Figures 7 and 8 about here

\section{SWS/PS-active neurons}

Three hundred and twenty-one of the 552 sleep-active neurons $(36.8 \%)$ showed a higher rate of spontaneous discharge during PS than during SWS (Table 1). Of these, 227 displayed no, or virtually no, spontaneous discharge during W (Figs. 3B and 9) and are hereafter referred to as "SWS/PS-specific" neurons. Like the SWS-specific neurons, the SWS/PS-specific neurons could be classified into fast (45/227) and slow $(182 / 227)$ groups, discharging, respectively, rapidly $(\geq 6 \mathrm{~Hz} ; \mathrm{n}=45)$ or slowly $(<6 \mathrm{~Hz} ; \mathrm{n}=182)$ during SWS (Fig. 3B). Of the 182 slow SWS/PS-specific neurons, 18 were referred to as being "PS-specific", because their spontaneous firing rates during PS were at least 4 times as high as during SWS and they discharged tonically throughout PS episodes (Fig. 10). The mean spontaneous firing rates of the 164 SWS/PS-specific and 18 PSspecific neurons across the wake-sleep cycle are shown in Fig. 3B and Fig. 3C, respectively. As shown in Fig. 1 (f-j), they were co-distributed with SWS-specific neurons.

Spike shape and spontaneous discharge rate and pattern of SWS/PS-specific neurons

Most of these neurons had a biphasic action potential and narrow spike shape. The mean spike duration $( \pm \mathrm{SD})$ of the slow and fast SWS/PS-specific neurons was $0.57 \pm 0.10 \mathrm{~ms}$ and $0.54 \pm 0.12 \mathrm{~ms}$, respectively, the difference not being statistically significant $(P>0.05$, two-tailed t test).

As shown in Fig. 3B, the activity of these two groups of SWS/PS-specific neurons was state-dependent in the order PS $>$ SWS $>$ D $>$ W. The difference in firing rate across the wake-sleep states was highly statistically 
significant in each group $(P<0.0001$, Kruskal-Wallis), as was the difference in firing rate between two successive sleep states in each group ( $P<0.01$, Mann-Whitney). As shown in Fig. 9, most of the SWS/PSspecific neurons fired either in single isolated spikes or in clusters (peak IMEANFR $<100 \mathrm{~Hz}$ ), whereas 6 of the 45 fast SWS/PS-specific and 11 of the 164 slow SWS/PS-specific neurons displayed high frequency burst discharges (peak IMEANFR $>150 \mathrm{~Hz}$; Fig. 13).

Insert Figures 9 and 10 about here

Activity profiles of SWS/PS-specific neurons during wake-sleep state transitions

At the transition from SWS to W, all SWS/PS-specific neurons ceased firing before the onset of EEG activation (Figs. 9 and 13). The mean $( \pm$ SD) interval between the last spike discharge and the onset of EEG activation was $189.7 \pm 106.7 \mathrm{~ms}$ for the fast SWS/PS-specific neurons. SWS/PS-specific neurons also ceased firing when waking was elicited during sleep by an arousing sound stimulus (Fig. 6). The mean ( \pm SD) interval between the last unit discharge and the onset of the stimulus was $-33.6 \pm 62.7 \mathrm{~ms}$ for the 14 fast SWS/PSspecific neurons tested.

During the transition from W to D, all the fast SWS/PS-specific neurons discharged after the onset of EEG synchronization (deactivation), with a mean $( \pm$ SD) latency of $1023.6 \pm 961.7 \mathrm{~ms}$ (Fig. 9). The mean time course of the unit discharge at the transitions from SWS to W and from W to D was estimated for the 4 most characteristic fast SWS/PS-specific neurons that fired at high rates at sleep onset. At the transition from SWS to W (Fig. 7, upper panel), the activity of the SWS/PS-specific neurons decreased significantly $0.1 \mathrm{~s}$ before the onset of W, as seen with SWS-specific neurons, while, at the transition from W to D (Fig. 7, lower panel), they fired $0.1 \mathrm{~s}$ after the onset of D and $0.2 \mathrm{~s}$ after the onset of unit activity of SWS-specific neurons. During the transition from PS to W, all the fast SWS/PS-specific neurons ceased firing before the end of PS, defined by the end of continuous rhythmic theta waves, with a mean $( \pm$ SD) interval of $138.9 \pm 116.5 \mathrm{~ms}$ (Fig. 9).

\section{PS-specific neurons}

Spike shape and spontaneous discharge rate and pattern of PS-specific neurons

PS-specific neurons had a biphasic action potential with a mean $( \pm \mathrm{SD})$ duration of $0.57 \pm 0.09 \mathrm{~ms}$, and fired essentially in single isolated spikes. As shown in Figs. 3C and 10, they exhibited a tonic discharge that was highly specific for PS.

Activity profiles of PS-specific neurons during wake-sleep state transitions 
Because of their very low unit activity during SWS, the activity profiles at the transitions from $\mathrm{W}$ to SWS and from SWS to W were not examined in detail. Without exception, however, they fired after the onset of D and ceased firing during SWS before the onset of W. During the transition from PS to W, they ceased firing before the end of rhythmic theta waves, with a mean $( \pm$ SD) interval of $274.3 \pm 235.3 \mathrm{~ms}(\mathrm{n}=22)$ (Fig. 10).

\section{Low rate sleep-specific neurons}

One hundred and thirty-seven of the $552(15.7 \%)$ sleep-active neurons were characterized by a very low unit activity $(<1.0 \mathrm{~Hz})$ which was specific to SWS and PS (Table 1) and were termed "low rate sleepspecific" neurons. The vast majority $(127 / 137)$ had a biphasic action potential and their overall mean $( \pm$ SD) spike duration was $0.58 \pm 0.09 \mathrm{~ms}$, not statistically different from that of the slow SWS/PS-specific neurons $(P>$ 0.05, two-tailed $t$ test). They were distributed evenly throughout the POA and BFB (Fig. 1, f-j).

Insert Figures 11 and 12 about here

\section{Waking-active neurons}

Waking-specific neurons. Of the 96 waking-active neurons, 49 (51.0\%) are referred to as "waking-specific (Wspecific)" neurons, because of their highly specific unit activity during W (Fig. 11). As with sleep-active neurons, they could be divided into fast $(\geq 9 \mathrm{~Hz} ; \mathrm{n}=21)$ and slow $(<9 \mathrm{~Hz} ; \mathrm{n}=28)$ groups (Fig. 12A). As shown in Fig. 1 (k-o), most of the $\mathrm{W}$-specific neurons were found in the ventral and middle region of the POA and BFB, including the LPO, vlPOA, and HDB. In contrast, they were essentially absent in the MPO.

Spike shape and spontaneous discharge rate and pattern of W-specific neurons

Except for two slow W-specific neurons, the W-specific neurons had a biphasic action potential. The mean $( \pm \mathrm{SD})$ spike durations of the slow and fast W-specific neurons were $0.62 \pm 0.24 \mathrm{~ms}$ and $0.50 \pm 0.07 \mathrm{~ms}$, respectively, the difference being statistically significant $(P<0.05$, two-tailed $t$ test). The mean spontaneous discharge rate of these two groups of W-specific neurons is illustrated in Fig. 12A. They fired at the highest rate during W, decreased their firing rate during D, and were virtually silent during both SWS and PS. The difference between the discharge rate during QW and that during D was statistically significant, as was the difference between D and SWS $(P<0.05$, Mann-Whitney). In contrast, the difference in firing rate between AW and QW was not $(P>0.05$, Mann-Whitney). Seven of the 21 slow W-specific neurons and 16 of the 28 fast W-specific neurons discharged mostly in single isolated spikes, while the remainder fired either in clusters (peak IMEANFR $<100 \mathrm{~Hz}, \mathrm{n}=7$ ) (Fig. 11) or in bursts (peak IMEANFR $>150 \mathrm{~Hz}, \mathrm{n}=5$ ) (Fig. 13). 
Activity profiles of the fast $W$-specific neurons during wake-sleep state transitions

During the transition from SWS to W, the fast W-specific neurons fired before the onset of EEG activation (Figs. 5A-2 and 11) with a mean ( \pm SD) interval of $267.0 \pm 128.7 \mathrm{~ms}$, in sharp contrast to HA wakingspecific neurons in the PH, which fired after waking onset (Fig. 7, upper panel; see also Takahashi et al., 2006). When waking was elicited during sleep by an arousing sound stimulus (Fig. 6), they responded in single isolated spikes to the stimulus with a mean $( \pm \mathrm{SD})$ latency of $135.9 \pm 164.2 \mathrm{~ms}(\mathrm{n}=7)$ (Fig. 6).

During the transition from $\mathrm{W}$ to $\mathrm{D}, \mathrm{W}$-specific neurons decreased their spontaneous firing rate or stopped firing concomitant with the onset of EEG synchronization (Figs. 5B-2 and 11), as shown by the presence of low spontaneous discharge activity during D (Fig. 12A). When the change in firing rate during the transition from $\mathrm{W}$ to $\mathrm{D}$ was compared to the reference (steady waking), W-specific neurons showed a significant decrease in discharge rate as early as $0.8 \mathrm{~s}$ before the onset of $\mathrm{D}$ (Fig. 7, lower panel). The unit activity of the W-specific neurons across the sleep-waking states was, therefore, inversely related to that of the SWS/PS-specific neurons. This relationship between the unit activities was clearly seen indirectly in separate recordings of W-specific and SWS/PS-specific neurons and directly when W-specific and SWS/PS-specific neurons were recorded simultaneously using the same electrode (Fig. 13).

\section{W/PS-active neurons}

$T I-S$ and TI-R neurons. W/PS-active neurons also consisted of slowly discharging $(<10 \mathrm{~Hz} ; \mathrm{n}=60)$ and rapidly discharging ( $\geq 10 \mathrm{~Hz} ; \mathrm{n}=46$ ) groups, referred to as "Type I-S (TI-S)" and "Type I-R (TI-R)", respectively (Fig. 12B and C). Twenty-six TI-S and 10 TI-R neurons discharged specifically during EEG activation occurring during W and PS, and were therefore termed "TI-Ss" and "TI-Rs", respectively. As shown in Fig. 1 (k-o), these W/PS-specific neurons were mainly located in the HDB and adjacent vlPOA, the regions containing cholinergic neurons (Rye et al., 1984; Tago et al., 1987), whereas the W/PS-active TI-S and TI-R neurons were distributed throughout the POA and BFB.

Insert Figures 13 and 14 about here

Spike shape and spontaneous discharge rate and pattern of TI-S neurons

W/PS-active TI-S neurons had a biphasic action potential, whereas W/PS-specific TI-Ss neurons had a broad triphasic action potential, characterized by a shoulder on the early descending phase of the spike potential 
(Figs. 14B and 17). The mean ( $\pm \mathrm{SD}$ ) spike duration of the TI-S and TI-Ss neurons was $0.53 \pm 0.07 \mathrm{~ms}$ and 0.86 $\pm 0.28 \mathrm{~ms}$, respectively, the difference being highly statistically significant $(P<0.001$, two-tailed $\mathrm{t}$ test).

The activity of TI-S and TI-Ss neurons was highest during both AW and PS, the difference in firing rate between these two states not being statistically significant $(P>0.05$, Mann-Whitney). During waking states, the mean firing rate of TI-Ss neurons was significantly higher during AW than during QW $(P<0.05$, MannWhitney), but no such difference was seen for TI-S neurons ( $P>0.05$, Mann-Whitney) (Fig. 12B).

Ten of the 34 W/PS-active TI-S neurons fired in single isolated spikes, the remainder discharging in clusters (peak IMEANFR < $100 \mathrm{~Hz}$ ). In sharp contrast, all W/PS-specific TI-Ss neurons displayed, during both W and PS, a single spike, low-frequency $(<10 \mathrm{~Hz})$ tonic firing pattern (Fig. 14A). The mean $( \pm \mathrm{SD}) \mathrm{CVs}$ for the spike interval during AW, QW, and PS were $0.82 \pm 0.23,0.81 \pm 0.19$, and $0.67 \pm 0.03$, respectively. The CV for the spike interval during PS was significantly smaller than that during waking $(P<0.01$, two-tailed t-test), indicating more regular firing in PS than in W.

Activity profiles of TI-Ss neurons during wake-sleep state transitions

During the transition from SWS to W, TI-Ss neurons displayed firing before the onset of EEG activation (Figs. 7, upper panel, and 14), with a mean $( \pm$ SD) interval of $281.5 \pm 119.4$ ms. During sleep, they responded to an arousing sound stimulus in single isolated spikes with a mean $( \pm \mathrm{SD})$ latency of $98.0 \pm 107.0 \mathrm{~ms}$ ( $n=19$ ) (Fig. 6). During the transition from W to D, they showed a significant decrease in spontaneous firing rate or completely stopped firing concomitant with the onset of EEG synchronization (Figs. 12B and 14), as seen with the fast $\mathrm{W}$-specific neurons. When the time course of unit discharge from $\mathrm{W}$ to $\mathrm{D}$ was estimated statistically, the TI-Ss neurons showed a significant decrease in discharge rate $0.6 \mathrm{~s}$ before the onset of D (Fig. 7, lower panel). When the time course of unit discharge at the transition from SWS to PS was estimated with 0.5 -s bins (n = 26), the TI-Ss neurons showed a significant increase in discharge rate $1.0 \mathrm{~s}$ before the onset of PS (Figs. $5 \mathrm{C}-2$ and $8 \mathrm{~A}-2$ and $\mathrm{B})$.

Spike shape and spontaneous discharge rate and pattern of TI-R neurons

All but 2 of the 36 W/PS-active TI-R neurons had a biphasic action potential and narrow spike shape, the other 2 showing a triphasic action potential with the same spike duration. The mean $( \pm \mathrm{SD})$ spike duration for all 36 was $0.51 \pm 0.09 \mathrm{~ms}$. All 10 W/PS-specific TI-Rs neurons also had a biphasic action potential, but a very narrow spike shape (Fig. 14C), their mean ( \pm SD) spike duration being $0.44 \pm 0.08 \mathrm{~ms}$, significantly shorter than that of the TI-R neurons ( $P<0.05$, two-tailed $\mathrm{t}$ test). Both TI-R and TI-Rs neurons fired either in single isolated spikes or in clusters (peak IMEANFR < $100 \mathrm{~Hz}$ ) during W and in either clusters or bursts during PS (Fig. 15). 
These high frequency rhythmic discharges were synchronized with rhythmic theta waves in all 10 TI-Rs neurons (Fig. 16) and in 3 of the 36 TI-R neurons. Like the TI-Rs neurons, these theta-related TI-R neurons were located in $(\mathrm{n}=2)$, or immediately adjacent to $(\mathrm{n}=1)$, the HDB (Fig. $1 \mathrm{k}, \mathrm{m}$, and o).

Activity profiles of TI-Rs neurons during wake-sleep state transitions

During the transition from SWS to W, all TI-Rs neurons discharged prior to the onset of EEG activation with a mean ( \pm SD) interval of $220.7 \pm 126.1 \mathrm{~ms}$ (Fig. 15). They responded to an arousing sound stimulus in single isolated spikes with a mean ( \pm SD) latency of $116.3 \pm 117.4 \mathrm{~ms}$ (Fig. 6). The mean time course of unit discharge at the transitions from SWS to W and from W to D was obtained from 4 of the most characteristic TIRs neurons and is shown in Fig. 7. As seen in this figure, the TI-Rs neurons displayed a sharp increase in discharge rate at waking onset (upper trace) and a significant decrease in firing rate $0.5 \mathrm{~s}$ prior to sleep onset (lower trace). At the transition from SWS to PS, they showed a significant increase in firing rate $0.5 \mathrm{~s}$ before the onset of PS and $1.5 \mathrm{~s}$ before the significant decrease in discharge rate of the SWS-specific neurons (Fig. 8A-3 and $B)$.

Insert Figures 15 and 16 about here

\section{Responses of POA and BFB neurons to electrical stimulation of the medial frontal cortex}

The effect of electrical stimulation of the medial frontal cortex was tested in a total of 174 POA and BFB neurons recorded ipsilaterally to the stimulation site. Of these, 2 showed antidromic responses (Fig. 17), while 47 exhibited excitatory synaptic responses (Fig. 18A and B), 5 inhibitory synaptic responses (Fig. 18C), and 120 no significant responses.

Antidromic responses. Antidromic responses were only elicited in 2 units, both of which were TI-Ss, characterized by a triphasic action potential and a location in the cholinergic region of the vlPOA (Fig. 17). Their latencies were 6.3 and $7.0 \mathrm{~ms}$, respectively, the axonal conduction velocities being $<1 \mathrm{~m} / \mathrm{s}$, indicative of finely myelinated axons. Further 6 TI-Ss neurons and 9 of the 11 TI-S neurons tested displayed no responses, while another TI-Ss and the other 2 TI-S neurons were activated orthodromically, with latencies varying between 4 and $30 \mathrm{~ms}$, as was the case for sleep-active and waking-active neurons (see below).

Insert Figures 17 and 18 about here 
Orthodromic responses. As seen with some TI-S and TI-Ss neurons, a significant number of sleep-active (36 of 116; $31 \%$ ) and waking-active ( 4 of $22 ; 18 \%$ ) neurons displayed excitatory synaptic responses either in single isolated spikes (Fig. 18A) or in bursts (Fig. 18B). The 36 sleep-active neurons consisted of 4 of the 14 SWSactive, 5 of the 19 LR SWS/PS-specific, 8 of the 14 SWS/PS-active, and 19 of the 69 SWS/PS-specific neurons tested. The 4 waking-active neurons consisted of 3 of the $8 \mathrm{~W}$-active and 1 of the $14 \mathrm{~W}$-specific neurons tested. A weak inhibitory synaptic response was only seen in one of the 14 SWS/PS-active, one of the 69 SWS/PSspecific (Fig. 18C), one of the $14 \mathrm{~W}$-specific, and 3 of the 13 TI-R neurons tested.

\section{Discussion}

Based on extracellular single unit recordings of 872 neurons across the complete sleep-waking cycle in the POA and BFB of mice, we have been able to describe the properties and distribution of particular groups of state-specific neurons, i.e. SWS-specific, SWS/PS-specific, PS-specific, W-specific, and W/PS-specific neurons, which might play an important role in the induction and maintenance of sleep and waking, on the one hand, and the activation and deactivation of cortical activity on the other, as discussed below.

\section{SWS-specific and SWS/PS-specific neurons as sleep-promoting neuronal populations}

Early single unit recording studies in the POA and BFB of cats revealed sleep-active neurons discharging at the highest rate during SWS (SWS discharge rate $>2$ times waking rate and active PS rate) (Szymusiak and McGinty, 1986; Szymusiak and McGinty, 1989). These were located in the HDB and LPO/SI region and some were reported to fire just prior to the appearance of the first high-amplitude spindle bursts in the EEG, a characteristic of light SWS (Szymusiak and McGinty, 1986). However, these neurons showed little change in spontaneous discharge rate during the transition from sleep to waking and thus differ from the SWSactive and SWS-specific neurons described in the present study. More recent studies in rats also reported the presence of a group of SWS-active neurons in the POA and BFB (Koyama and Hayaishi, 1994; Lee et al., 2004; Osaka and Matsumura, 1994). These were distributed throughout the POA and some were reported to fire before the onset of sleep (Koyama and Hayaishi, 1994). However, the presence of spontaneous discharges during waking in these previous studies in cats and rats make it difficult to determine precisely their activity profiles at the state transitions from waking to sleep and from sleep to waking.

In the present study in mice, 11 of the 25 fast SWS-specific neurons discharged at the transition from waking to sleep, prior to the onset of EEG synchronization, the first sign of EEG sleep. All the fast SWS-specific neurons displayed high rates of spontaneous discharge as early as sleep onset (drowsy state) and maintained high 
frequency unit activity during SWS, while they either showed a significant decrease in firing rate in PS or ceased firing before waking onset. These findings suggest that the SWS-specific neurons may play an important role in the processes of sleep onset and sleep maintenance, and could therefore function as sleep-promoting neurons. The SWS/PS-specific neurons might also be sleep-promoting neural elements within the POA and BFB, although their discharge did not anticipate the onset of sleep and they fired at the highest rate during PS. They were the most commonly encountered cells and many fired at a high rate at sleep onset, increased their discharge rate as the degree of cortical synchrony increased, and stopped firing before waking onset. They may therefore play a role not in the induction, but in the enhancement and maintenance of sleep.

In the present study, SWS-specific neurons were shown to be located in the HDB and adjacent vlPOA at the rostral level of recording sites (Fig. $1 \mathrm{a}$ and b), but to be more widely distributed in the lateral MPO just adjacent to the LPO, LPO, LH, SI, and AHA at the middle and caudal levels (Fig. 1, f-j). SWS/PS-specific neurons, including those with a very low firing rate, were also found diffusely in the POA and BFB. These findings are in general agreement with those of early and recent studies in cats (Szymusiak and McGinty, 1986) and rats (Koyama and Hayaishi, 1994; Lee et al., 2004; Osaka and Matsumura, 1994, 1995; Suntsova et al., 2002). However, it should be mentioned that the MPO was largely devoid of fast SWS/PS-specific and SWSspecific neurons, both of which may be primary sleep-promoting elements, and that these sleep-specific neurons tended to be localized in the lateral and ventral POA and adjacent BFB structures, such as the HDB, vlPOA, and ventral part of the LPO and LH, the sites in which W-specific and W/PS-specific neurons were also concentrated (Fig. 1, k-o).

Recently, based on the sleep-related expression of the immediate early gene, c-fos, a restricted collection of neurons has been described in the rat ventrolateral POA, designated as the ventrolateral preoptic nucleus (VLPO, corresponding to the vIPOA in the present account in mice), and were proposed to be the principal sleep-promoting cell group within the POA and BFB (Sherin et al., 1996). Moreover, using the same method, the same group of authors proposed that a region surrounding the VLPO (called the extended VLPO) might be the critical PS-promoting cell group (Lu et al., 2000). A single unit recording study in rats reported that sleep-active neurons are indeed localized in the VLPO (Szymusiak et al., 1998); however, this result could only be applied to the most rostral region of the LPO, the only preoptic region in which recordings were made by the authors (see above). In the present study, we revealed the presence of many PS-specific neurons, which showed a high rate of tonic discharge confined to a PS episode. These neurons were found throughout the POA and LH, this finding again being at variance with the assumption that the critical PS-promoting cell group is located in a 
restricted region of the POA (Lu et al., 2000). In addition, within the ventral POA and BFB areas, there was considerable overlap between the sites at which SWS-specific, SWS/PS-specific, PS-specific, W-specific, and W/PS-specific units were recorded. These findings, therefore, do not support the view that particular clusters of neurons localized in the ventrolateral region of the POA play a critical role in the induction and maintenance of sleep, including PS, but are in line with the assumption that sleep-promoting hypnogenic neurons are distributed throughout the POA and adjacent BFB and that the size of lesion within the POA and BFB determines the magnitude of sleep deficit induced by the lesion (McGinty and Szymusiak, 2001). Indeed, lesions including the dorsal or lateral LPO, but sparing the VLPO, cause severe insomnia in rats (Lu et al., 2000; Schmidt et al., 2000) and the effects of lesions on sleep in general depend on the size or extension of the lesion in both rats and cats (Nauta, 1946; Sallanon et al., 1989)(see also McGinty and Szymusiak, 2001 for review). In addition, muscimol, a potent GABA agonist, induces severe insomnia when microinjected into any region of the LPO in the cat (Lin et al., 1989; Sakai et al., 1990). In this regard, it should be noted that both nonselective (McGinty and Sterman, 1968; Nauta, 1946) and cell-selective (Sallanon et a1., 1989; Schmidt et a1., 2000; Szymusiak and McGinty, 1986) neurotoxic lesions of the LPOA and adjacent BFB result in robust suppression of both deep SWS and PS in the rat and cat, whereas lesions of the MPO alone have little or no effect in the rat (Asala et al., 1990; Schmidt et al., 2000), suggesting that the LPO plays a primary role in the mechanisms of sleep-waking regulation within the POA. The present findings that fast SWS-specific and SWS/PS-specific neurons are virtually absent in the MPO may corroborate those from these lesion studies.

A recent in vivo study in anesthetized rats revealed several populations of GABAergic neurons in the BFB just adjacent to the LPO: $40 \%$ of these GABAergic neurons showed an increase in discharge rate on somatosensory-evoked cortical activation, while the remaining $60 \%$ showed a decrease in discharge rate on cortical activation and fired in either clusters or bursts during cortical deactivation (Mannes et al., 2000). The latter group might represent the SWS-active and/or SWS/PS-active neurons described previously in rats and cats (Koyama and Hayaishi, 1994; Szymusiak and McGinty, 1986) and in the present study in mice. They may also correspond to populations of GABAergic neurons that express c-fos following sleep recovery after sleep deprivation (Gvilia et al., 2006; Modirrousta et al., 2004).

\section{Waking-specific neurons as a waking-promoting neuronal population}

W-specific neurons fired at the highest rate during waking, displayed a sharp decrease in firing rate during D, and became silent during both SWS and PS, as do the Orx/Hcrt- and non-Orx/Hcrt waking-active neurons that we have recently reported in the PH of mice (Takahashi et al., 2008). As also seen with the PH 
waking-active neurons, the POA/BFB W-specific neurons fired before the onset of $\mathrm{W}$, with a sharp increase in discharge rate. These data suggest that the POA/BFB W-specific neurons may be waking-promoting neural elements in the forebrain. Several lines of evidence point to important differences between the putative wakingpromoting neurons in the $\mathrm{PH}$ and those in the POA/BFB; i) the majority of the POA/BFB W-specific neurons displayed higher discharge rates $(>10 \mathrm{~Hz})$ than the $\mathrm{PH}$ waking-active neurons $(<10 \mathrm{~Hz})$; ii) at the transition from SWS to W, the POA/BFB W-specific neurons fired before the onset of EEG activation with a mean $( \pm \mathrm{SD})$ interval of $267.0 \pm 128.7 \mathrm{~ms}$, while Orx/Hcrt- and non-Orv/Hcrt waking-active neurons fire with significantly shorter mean intervals of $141.5 \pm 82.3 \mathrm{~ms}$ and $108.5 \pm 57.7 \mathrm{~ms}$, respectively; and iii) the $\mathrm{PH}$ waking-active neurons respond in clusters to an arousing sound stimulus with a short latency $(\approx 10 \mathrm{~ms})$, while the POA/BFB W-specific neurons responded in single isolated spikes with a longer latency (Takahashi et al., 2008 for the PH waking-active neurons and the present study for the POA/BFB waking-specific neurons). These data suggest differences in the excitatory or inhibitory inputs impinging on the PH and POA/BFB waking-promoting neurons and a different role in the mechanisms of waking. In any case, the activation or inactivation of these wakingpromoting neurons at state transitions does not seem to result from cessation (disinhibition) or activation (inhibition) of sleep-promoting neuronal activity, because significant changes in the activity of the sleeppromoting neurons during the state transitions followed, and did not precede, that of the waking-promoting neurons (Fig. 7) (see below). Our data therefore refute the prevailing hypothesis that the sleep process starts with the activation of the POA/BFB hypnogenic neurons (Jones, 2005; McGinty and Szymusiak, 2001; Sakai and Crochet, 2003; Saper et al., 2001). The exact neurochemical phenotype of these waking-specific neurons is unknown. However, it is worth mentioning that the spike shape and firing properties of the waking-specific neurons resemble those of identified glutamatergic neurons in the rat BFB (Mannes et al., 2003), suggesting their glutamatergic nature.

Insert Figure19 about here

\section{Role of TI-Ss and TI-Rs neurons in the process of cortical activation during waking and PS}

In the present study, we found two populations of W/PS-specific neurons that fired specifically during waking and PS in association with cortical activation, namely the TI-Ss and TI-Rs neurons. TI-Ss neurons displayed a slow tonic isolated discharge pattern during both W and PS, as seen with a cluster of ascending mesopontine cholinergic neurons in rats and cats (E1 Mansari et al., 1989; Koyama et al., 1998; Koyama and 
Sakai, 2000; Steriade et al., 1990). In contrast, TI-Rs neurons fired either in clusters or in bursts during W and in high frequency bursts during PS in close association with cortical theta waves. The unit activity of both types of neurons was highest during AW and PS, the difference in firing rate between these two states not being statistically significant. Moreover, both discharged at a high rate during QW without motor activity, fired before the onset of waking at the transition from sleep, and stopped firing concomitant with the onset of EEG synchronization, as seen with W-specific neurons (Fig. 7). These findings strongly suggest an important role of these W/PS-specific neurons in the processes of cortical activation/deactivation. The TI-Ss and TI-Rs W/PSspecific neurons were localized in the HDB and adjacent vIPOA, the regions containing cholinergic neurons sending axons to the cortex (Armstrong et al., 1983; Ichikawa et a1., 1997; Rye et a1., 1984; Tago et al., 1987), whereas both TI-S and TI-R neurons were widely distributed in noncholinergic regions of the POA/BFB (Fig. 1, k-0). Furthermore, 2 of the 8 TI-Ss neurons tested were activated antidromically by stimulation of the medial frontal cortex with a long latency, indicating that they are cortically projecting neurons with a slowly conducting axon. These findings are in line with those from anatomical studies in the rat showing that cholinergic projections to the medial cortical areas arise from neurons located in the medial extent of the HDB (Rye et al., 1984; Saper, 1984). The TI-Rs neurons had a biphasic narrow action potential, whereas the TI-Ss neurons were characterized by a triphasic broad action potential, another characteristic of cholinergic neurons. The TI-Ss neurons, therefore, appear to be cholinergic neurons. In this regard, previous in vitro studies in guinea-pig BFB slices showed that chemically identified nucleus basalis cholinergic neurons display both a slow tonic $(<20 \mathrm{~Hz})$ discharge mode and a high frequency bursting $(>100 \mathrm{~Hz})$ mode, the latter being mediated by a calciumdependent low threshold spike (LTS) (Alonso et al., 1996; Khateb et a1., 1992). Moreover, recent in vivo studies in rats showed that identified BFB cholinergic neurons have a long spike duration and display robust rhythmic bursting activity in association with cortical activation (Lee et a1., 2005; Mannes et a1., 2000). In unanesthetized rats, identified BFB cholinergic neurons are reported to discharge in bursts of spikes during both AW and PS and to discharge at the highest rate during PS, at an intermediate rate during AW, and at a low rate during QW without motor activity (Lee et al., 2005). In the present study in mice, rhythmic bursting activity in association with cortical activation was seen in TI-Rs neurons, which have a narrow action potential, but never in TI-Ss neurons, which have a broad action potential. The unit activity of both TI-Ss and TI-Rs neurons was highest during AW and PS, and both discharged at a high rate during QW without motor activity. These findings indicate that both TI-Ss and TI-Rs differ from the identified cholinergic neurons reported in the rat BFB (Lee et al., 2005). 
In a recent study in urethan-anesthetized rats, Duque et al. (Duque et al., 2000) reported that identified BFB cholinergic neurons do not show any robust bursting during spontaneous or stimulation-induced EEG activation, suggesting that high frequency bursting is not a unique feature of BFB cholinergic neurons. Moreover, no high frequency bursting cells in association with cortical activation were found in the cholinergic BFB in cats (Détari et al., 1984; Détari et al., 1997; Szymusiak and McGinty, 2000) and, currently, nothing is known about the unit activity of identified cholinergic BFB neurons in mice. These findings, therefore, do not rule out the possibility that TI-Ss neurons are cholinergic in mice. Future studies using electrophysiological and chemical identification of recorded cells are necessary to test whether this is the case.

\section{Sleep-waking switch: disfacilitatory and inhibitory mechanisms}

In the POA/BFB, we demonstrated the presence of sleep-specific, waking-specific, and cortical activation-specific neurons that might be good candidates for, respectively, sleep-promoting, waking-promoting, or cortical activation-promoting neurons. There was an inverse relationship between the unit activity of the SWS-specific neurons and that of the W/PS-specific neurons and between that of SWS/PS-specific and Wspecific neurons (Figs. 13 and 19). Osaka and Matsumura (Osaka and Matsumura, 1994, 1995) reported that, in unanesthetized rats, POA/BFB sleep-active neurons are inhibited by applied noradrenaline (NA) or electrical stimulation of brainstem catecholaminergic neurons, the effect being mediated by activation of $\alpha_{2}$ adrenoceptors, while, in sharp contrast, POA/BFB waking-active neurons are excited by NA via activation of $\alpha_{1}$ adrenoceptors. In an in vitro study in rat brain slices, Gallopin et al. (Gallopin et al., 2000) reported a homogeneous group of presumed GABAergic sleep-promoting neurons in the vlPOA which show an LTS crowned by a high frequency burst of action potentials and are inhibited by NA, acetylcholine, and serotonin, but are unaffected by HA. The LPO and BFB receive dense noradrenergic and serotonergic, but only scarce cholinergic, projections from the brainstem (Jones and Cuello, 1989), suggesting that the cholinergic afferent projections may originate in local BFB cholinergic neurons. TI-Ss neurons might represent potential cholinergic neural elements in the mouse.

As shown in Fig. 19, we hypothesize that both SWS-specific (SWS-on) and SWS/PS-specific (SWS/PSon) neurons are GABAergic, while W/PS-specific (W/PS-on) neurons, in particular TI-Ss neurons, are cholinergic (ACh) and that there is a reciprocal inhibitory interaction between the SWS-on and W/PS-on (TI-Ss) neurons. The GABAergic SWS/PS-on neurons inhibit possibly glutamatergic (Glu) W-specific (W-on) neurons, which in turn excite the W/PS-on neurons. Both the POA/BFB SWS-on and SWS/PS-on neurons receive inhibitory inputs from the brainstem noradrenergic (NA) and adrenergic (A) W-on neurons, while the POA/BFB W-on and W/PS-on neurons receive excitatory inputs from the brainstem catecholaminergic W-on neurons, as 
well as from the PH Orx/Hcrt and non-Orx/Hcrt waking-active and HA waking-specific W-on neurons, all of which are, in turn, under the inhibitory control of the sleep-promoting POA/BFB SWS-on and SWS/PS-on neurons. The POA/BFB W/PS-on neurons receive an additional excitatory input from presumed glutamatergic brainstem PS-on neurons (Sakai et al., 2001). The SWS-on and SWS/PS-on neurons function as sleep-promoting neurons, while all the W-on and W/PS-on neurons function as waking-promoting neurons.

At the transition from waking to sleeping (Fig. 7, lower panel), the waking-promoting neurons either ceased firing (HA W-on neurons) or showed a significant decrease in firing rate (POA/BFB W-on and W/PS-on neurons, as well as Orx- and non-Orx W-on neurons; see Takahashi et al., 2008) before the onset of activity of the sleep-promoting neurons, indicating that the sleep process does not start with activation of the forebrain sleep-promoting neurons, but starts with deactivation (disfacilitation) of the waking-promoting neurons which results in disinhibition of the sleep-promoting neurons, thereby enhancing the sleep process. At the transition from sleeping to waking (Fig. 7, upper panel), the waking process starts with excitation of the waking-promoting neurons, leading to inhibition of the sleep-promoting neurons, thereby reinforcing the waking process. A key question is the identity of the critical waking-promoting neurons that control the sleep-waking switch. It is well known that sleep results from complex interactions between multiple waking-promoting and sleep-promoting systems distributed throughout the brain (Jones, 2005; Sakai and Crochet, 2003). However, it is important to mention that the POA/BFB waking-promoting neurons are under powerful excitatory adrenergic and noradrenergic control (Osaka and Matsumura, 1994, 1995), whereas the waking-specific HA and waking-active Orx/Hcrt neurons are, respectively, unaffected or inhibited by these inputs (Brown et al., 2001; Li et al., 2002; Yamanaka et a1., 2003). As shown in this and our previous studies (Takahashi et a1., 2006, 2008), both Orx/Hcrt and non-Ory/Hcrt waking-active neurons respond in clusters to an arousing sound stimulus with a short latency, but this was not the case for the POA/BFB waking-promoting neurons, which did, however, discharge before the firing of the PH waking-promoting neurons. It seems likely that neural activation of the $\mathrm{PH}$ waking-promoting neurons is largely due to external or internal excitatory stimuli, such as sensory, somatic, and visceral inputs, while that of the POA/BFB waking-promoting neurons is basically due to excitation of brainstem adrenergic and noradrenergic neurons. Detailed analysis of the unit activity at state transitions is needed for the brainstem catecholaminergic neurons in the mouse. This problem is currently under investigation in our laboratory. Finally, recent studies suggest that POA neurons play a role in the homeostatic aspects of sleep-waking regulation and are important targets for endogenous somnogens (reviewed in Szymusiak et al., 2007). Further studies are 
needed to determine whether some, or all, of the groups of state-specific neurons revealed in the present study play a role in this homeostatic regulation of sleep and waking.

\section{Conclusions}

The present study reveals, for the first time in the POA and BFB in conscious mice, the presence of sleep-specific, waking-specific, and cortical activation-specific neurons that might play an important role in the induction and maintenance of sleep and waking and in the activation and deactivation of cortical activity. The present findings suggest (i) that the forebrain sleep/waking switch is regulated by the opposing activities of sleep-promoting (SWS-specific and SWS/PS-specific) and waking-promoting (W-specific and W/PS-specific) neurons, (ii) that initiation of sleep is caused by decreased activity (disfacilitation) of the waking-promoting neurons, which, in turn, disinhibit the activity of the sleep-promoting neurons, thereby enhancing the sleep process, and (iii) that the activity of W/PS-specific neurons is deeply involved in the processes involved in cortical activation/deactivation. 


\section{REFERENCES}

Alam MN, McGinty D, Szymusiak R (1996) Preoptic/anterior hypothalamic neurons: thermosensitivity in wakefulness and non rapid eye movement sleep. Brain Res 718:76-82.

Alonso A, Khateb A, Fort P, Jones B, Mühlethaler M (1996) Differential oscillatory properties of cholinergic and non-cholinergic nucleus basalis neurons in guinea pig brain slices. Eur J Neurosci 8:169-182.

Armstrong DA, Saper CB, Levey AI, Wainer BH, Terry RD (1983) Distribution of cholinergic neurons in the rat brain demonstrated by the immunohistochemical localization of choline acetyltransferase. J Comp Neurol $216: 53-68$.

Asala SA, Okano Y, Honda K, Inoue S (1990) Effects of medial preoptic lesions on sleep and wakefulness in unrestrained rats. Neurosci Res 114:300-304.

Boulant JA, Dean JB (1986) Temperature receptors in the central nervous system. Ann Rev Physiol 48:639-654.

Brown RE, Stevens DR, Haas HL (2001) The physiology of brain histamine. Prog Neurosbiol 63:637-672.

Détari L, Juhasz G, Kukorelli T (1984) Firing properties of cat basal forebrain neurons during sleep-wakefulness cycle. Electroenceph Clin Neurophysiol 58:362-368.

Détari L, Semba K, Rasmusson DD (1997) Responses of cortical EEG-related basal forebrain neurons to brainstem ans sensory stimulation in urethane-anaesthetized rats. 1997 9:1153-1161.

Duque A, Balatoni B, Detari L, Zaborszky L (2000) EEG correlation of the discharge properties of identified neurons in the basal forebrain. J Physiol 28:1627-1635.

El Mansari M, Sakai K, Jouvet M (1989) Unitary characteristics of presumptive cholinergic tegmental neurons during the sleep-waking cycle in freely moving cats. Exp Brain Res 76:519-529.

Everitt BJ (1990) Sexual motivation: a neural and behavioural analysis of the mechanisms underlying appetitive and copulatory responses of male rats. Neurosci Biobehav Rev 14:217-232.

Everitt BJ, Robbins TW (1997) Central cholinergic systems and cognition. Ann Rev Psychol 48:649-684.

Gallopin T, Fort P, Eggermann E, Cauli B, Luppi PH, Rossier J, Audinat E, Mühlethaler M, Serafin M (2000) Identification of sleep-promoting neurons in vitro. Nature 404:992-995.

Gong H, Szymusiak R, King J, Steininger T, McGinty D (2000) Sleep-related c-Fos expression in the preoptic hypothalamus: effects of ambient warming. Am J Physiol 279:R2079-R2088.

Gvilia I, Xu F, McGinty D, Szymusiak R (2006a) Homeostatic regulation of sleep: a role for preoptic area neurons. J Neurosci 26:9426-9433. 
Gvilia I, Turner A, McGinty D, Szymusiak R (2006b) Preoptic area neurons and the homeostatic regulation of rapid eye movement sleep. J Neurosci 26:3037-3044.

Huang ZL, Urade Y, Hayaishi O (2007) Prostaglandins and adenosine in the regulation of sleep and wakefulness. Curr Opin Pharmacol 7:33-38.

Ichikawa T, Ajiki K, Matsuura J, Misawa H (1997) Localization of two cholinergic markers, choline acetyltransferase and vesicular acetylcholine transporter in the central nervous system of the rat: in situ hybridization histochemistry and immunohistochemistry. J Chem Neuroanat 13:23-39.

Jones BE (2004) Activity, modulation and role of basal forebrain cholinergic neurons innervating the cerebral cortex. Prog Brain Res 145:157-169.

Jones BE (2005) Basic mechanisms of sleep-wake states. In: Principles and Practice of Sleep Medicine (Kryger MH, Roth T, Dement W, eds), pp136-153. Philadelphia: Elsevier Saunders.

Jones BE (2005) From waking to sleeping: neuronal and chemical substrates. Trends Pharmacol Sci 26:578-586.

Jones BE, Cuello AC (1989) Afferents to the basal forebrain cholinergic cell area from pontomesencephalic catecholamine, serotonin, and acetylcholine - neurons. Neuroscience 31:37-61.

Khateb A, Mühlethaler M, Alonso A, Serafin M, Mainville L, Jones BE (1992) Cholinergic nucleus basalis neurons display the capacity for rhythmic bursting activity mediated by low-threshould calcium spikes. Neuroscience 51:489-494.

Koyama Y, Hayaishi O (1994) Firing of neurons in the preoptic/anterior hypothalamic areas in rat: its possible involvement in slow wave sleep and paradoxical sleep. Neurosci Res 19:31-38.

Koyama Y, Honda T, Kusakabe M, Kayama Y, Sugiura Y (1998) In vivo electrophysiological distinction of histocemically-identified cholinergic neurons using extracellular recording and labeling in rat laterodorsal tegmental nucleus. Neuroscience 83:1105-1112.

Koyama Y, Sakai K (2000) Modulation of presumed cholinergic mesopontine tegmental neurons by acetylcholine and monoamines applied iontophoretically in unanesthetized cats. Neuroscience 96:723-733.

Lee MG, Hassani OK, Alonso A, Jones B (2005) Cholinergic basal forebrain neurons burst with theta during waking and paradoxical sleep. J Neurosci 25:4365-4369.

Lee MG, Manns ID, Alonso A, Jones BE (2004) Sleep-wake related discharge properties of basal forebrain neurons recorded with micropipette in head-fixed rats. J Neurophysiol 92:1182-1198. 
Li Y, Gao XB, Sakurai T, van den Pol AN (2002) Hypocretin/Orexin excites hypocretin neurons via a local glutamate neuron-A potential mechanism for orchestrating the hypothalamic arousal system. Neuron 36:1169-1181.

Lin JS (2000) Brain structures and mechanisms involved in the control of cortical activation and wakefulness, with emphasis on the posterior hypothalamus and histaminergic neurons. Sleep Med Rev 4:471-503.

Lin JS, Sakai K, Vanni-Mercier G, Jouvet M (1989) A critical role of the posterior hypothalamus in the mechanisms of wakefulness determined by microinjection of muscimol in freely moving cats. Brain Research 479:225-240.

Lu J, Greco M, Shiromani P, Saper C (2000) Effecy of lesions of the ventrolateral preoptic nucleus on NREM and REM sleep. J Neurosci 20:3830-3842.

Mannes ID, Alonso A, Jones B (2000) Discharging profiles of juxtacellularly labeled and immunohistochemically identified cholinergic basal forebrain neurons recorded in association with the electroencephalogram in anesthetized rats. J Neurosci 20:1505-1518.

Mannes ID, Alonso A, Jones B (2000) Discharging profiles of juxtacellularly labeled and immunohistochemically identified GABAergic basal forebrain neurons recorded in association with the electroencephalogram in anesthetized rats. J Neurosci 20:9252-9263.

Mannes ID, Alonso A, Jones B (2003) Rhythmically discharging basal forebrain units comprise cholinergic, GABAergic, and putative glutamatergic cells. J Neurophysiol 89:1057-1066.

McGinty D, Sterman MB (1968) Sleep suppression after basal forebrain lesions in the cat. Science 160:12531255.

McGinty D, Szymusiak R (2001) Brain structures and mechanisms involved in the generation of NREM sleep: focus on the preoptic hypothalamus. Sleep Med Rev 5:323-342.

Modirrousta M, Mainville L, Jones BE (2004) GABAergic neurons with alpha2-adrenergic receptors in basal forebrain and preoptic area express c-Fos during sleep. Neuroscience 129:803-810.

Moruzzi G (1972) The sleep-waking cycle. Ergeb Physiol 64:1-165.

Nauta WJH (1946) Hypothalamic regulation of sleep in rats. An experimental study. J Neurophysiol 9:285-316.

Osaka T, Matsumura H (1994) Noradrenergic inputs to sleep-related neurons in the preoptic area from the locus coeruleus and the ventrolateral medulla in the rat. Neurosci Res 19:39-50.

Osaka T, Matsumura H (1995) Noradrenaline inhibits preoptic sleep-active neurons through $\alpha_{2}$-receptors in the rat. Neurosci Res 21:323-330. 
Rye DB, Wainer BH, Mesulam MM, Mufson EJ, Saper CB (1984) Cortical projections arising from the basal forebrain: a study of cholinergic and non-cholinergic components employing combined retrograde tracing and immunohistological localization of choline acetyltransferase. Neuroscience 13:627-643.

Sakai K, Crochet S (2003) A neural mechanism of sleep and wakefulness. Sleep Biol Rhyth 1:29-42.

Sakai K, Crochet S, Onoe H (2001) Pontine structures and mechanisms involved in the generation of paradoxical (REM) sleep. Arch Ital Biol 139:93-107.

Sakai K, El Mansari M, Lin JS, Zhang JG, Vanni-Mercier G (1990) The posterior hypothalamus in the regulation of wakefulness and paradoxical sleep. In: The Diencephalon and Sleep (Mancia. M, Marini. M, eds), pp171198. New York: Raven Press.

Sakurai T (2002) Roles of orexins in regulation of feeding and wakefulness. NeuroReport 13:987-995.

Sallanon M, Denoyer M, Kitahama K, Jouvet M (1989) Long-lasting insomnia induced by preoptic neuron lesions and its transient reversal by muscimol injection into the posterior hypothalamus in the cat. Neuroscience 32:669-683.

Saper CB (1984) Organization of cerebral systems in the rat. I. Magnocellular basal nucleus. J Comp Neurol 222:313-342.

Saper CB, Chou TC, Scammell TE (2001) The sleep switch: hypothalamic control of sleep and wakefulness. Trends Neurosci 24:726-731.

Schmidt MH, Valatx J, Sakai K, Fort P, Jouvet M (2000) Role of the lateral preoptic area in sleep-related erectile mechanisms and sleep generation in the rat. J Neurosci 20:6640-6647.

Sherin JE, Shiromani PJ, McCarley RW, Saper CB (1996) Activation of ventrolateral preoptic neurons during sleep. Science 271:216-219.

Steriade M, Datta S, Paré D, Oakson G, Curró Dossi R (1990) Neuronal activities in brainstem cholinergic nuclei related to tonic activation processes in thalamocortical systems. J Neurosci 10:2541-2559.

Suntsova N, Szymusiak R, Alam M N, Guzman-Marin R, McGinty D (2002) Sleep-waking discharge patterns of median preoptic nucleus neurons in rats. J Physiol 543:665-677.

Szymusiak R, Alam N, Steininger TL, McGinty D (1998) Sleep-waking discharge patterns of ventrolateral preoptic/anterior hypothalamic neurons in rats. Brain Res 803:178-188.

Szymusiak R, McGinty D (1986) Sleep suppression following kainic acid-induced lesions of the basal forebrain. Exp Neurol 598-614. 
Szymusiak R, McGinty D (1986) Sleep-related neuronal discharge in the basal forebrain of cats. Brain Res $370: 82-92$

Szymusiak R, McGinty D (1989) Sleep-waking discharge of basal forebrain projection neurons in cats. Brain Res Bull 22:423-430.

Szymusiak R, McGinty D (2000) Discharge patterns of neurons in cholinergic regions of the basal forebrain during waking and sleep. Behav Brain Res 115:171-182.

Szymusiak R, Gvilia I, McGinty D (2007) Hypothalamic control of sleep. Sleep Med 8:291-301.

Tago H, McGeer PL, Bruce G, Hersh L (1987) Distribution of choline acetyltransferase-containing neurons of the hypothalamus. Brain Res 415:49-62.

Takahashi K, Lin JS, Sakai K (2006) Nuronal activity of histaminergic tuberomammillary neurons during wakesleep states in the mouse. J Neurosci 26:10292-10298.

Takahashi K, Lin JS, Sakai K (2008) Neuronal activity of orexin and non-orexin waking-active neurons during wake-sleep states in the mouse. Neuroscience 153:860-870.

von Economo C (1930) Sleep as a problem of localization. J Nerv Ment Dis 71:249-259.

Yamanaka A, Muraki Y, Tsujino N, Goto K, Sakurai T (2003) Regulation of orexin neurons by the monoaminergic and cholinergic systems. Biochem Biophysi Res Commun 303:120-129. 


\section{Figure legends}

Figure 1. Camera lucida drawings of frontal sections (five different planes at $0.2 \mathrm{~mm}$ intervals rostral to caudal). (a-e) Distribution of ChAT-immunoreactive neurons (dots and circles). (f-j) the four groups of sleep-active neurons (squares, circles, circles with a central dot, and dots). (k-0) Distribution of waking-specific (squares), waking/PS-specific (circles), and waking/PS-active (dots and thick-lined circles) neurons. The dots and circles in a-e indicate heavily and faintly stained ChAT-immunoreactive neurons, respectively (see Tago et al., 1987). The large and small symbols indicate rapidly firing (fast) and slowly firing (slow) neurons, respectively. The thicklined circles in $\mathrm{k}, \mathrm{m}$, and o indicate W/PS-active neurons discharging in close relation to theta waves. $3 \mathrm{~V}$, third ventricle; ac, anterior commissure; AHA, anterior hypothalamic area; BST, bed nucleus of the stria terminalis; f, fornix; HDB, horizontal limb of the diagonal band of Broca; LA, lateroanterior hypothalamic nucleus; LH, lateral hypothalamic area; LPO, lateral preoptic area; MCPO, magnocellular preoptic nucleus; MPO, medial preoptic area; opt, optic tract; OX, optic chiasma; SCh, suprachiasmatic nucleus; SI, substantia innominata; SON, supraoptic nucleus; VLPO, ventrolateral preoptic nucleus; vlPOA, ventrolateral preoptic area; VP, ventral pallidum. Nomenclatures according to Paxinos and Franklin, 2001. Note that VLPO refers to different structures in the mouse and the rat (see Gallopin et al., 2000; Sherin et al., 1996), the latter being equivalent to that referred to as the vIPOA in the present account.

Figure 2. Activity of a representative fast SWS-specific neuron. Note the high frequency burst firing during SWS and cessation of discharge during both waking and PS. The trace showing the bursting discharges is expanded and shown in the box. The arrows indicate the state transitions.

Figure 3. Mean spontaneous discharge rate of SWS-specific (A), SWS/PS-specific (B), and PS-specific (C) neurons across the wake-sleep cycle. The SWS-specific neurons were subdivided into 2 groups on the basis of their high $(\geq 9 \mathrm{~Hz})$ or low $(<9 \mathrm{~Hz})$ spontaneous firing rates during SWS. The SWS/PS-specific neurons were also subdivided into 2 groups on the basis of their high $(\geq 6 \mathrm{~Hz})$ or low $(<6 \mathrm{~Hz})$ spontaneous firing rates during SWS. Note that the difference in discharge rate across wake-sleep states was highly statistically significant $(P<0.0001$, Kruskal-Wallis) in each group. The differences between AW and QW in A and B and between D and SWS in A were not statistically significant ( $P>0.05$, Mann-Whitney), but all other differences between two successive states were significant $(P<0.05$, Mann-Whitney) in each group. 
Figure 4. Photomicrographs showing the distribution of ChAT-immunoreactive neurons and the unit recording sites marked with Pontamine Sky Blue (arrows). In a, the arrowheads indicate faintly labeled ChATimmunoreactive neurons, while the arrow indicates the recording site of a waking-specific neuron. The left and right arrows in $\mathrm{b}$ indicate the recording sites of an SWS-specific and an SWS/PS-specific neuron, respectively. HDB, horizontal limb of the diagonal band of Broca; LPO, lateral preoptic area; vlPOA, ventrolateral preoptic area.

Figure 5. Discharge pattern of SWS-specific (A1, B1, and C1), W-specific (A2 and B2), and TI-Ss (C2) neurons during wake-sleep state transitions.

The dashed lines indicate the state transitions. During the transitions from SWS to W (A) and W to D (B), onset of W was defined by the first sign of EEG desynchronization (activation), while sleep onset was defined by the first appearance of synchronized (deactivated) EEG. During the transition from SWS to PS (C), PS onset was defined by the appearance of continuous rhythmic theta waves on the cortical EEG.

Figure 6. Discharge pattern of the five groups of neurons in response to an arousing sound stimulus given during sleep. Note the suppression of discharge in the sleep-specific neurons and the excitatory response in the Wspecific and W/PS-specific neurons.

Figure 7. Mean time course of the discharge rate for the five groups of BFB/POA sleep-specific, W-specific, and W/PS-specific neurons and the group of TM W-specific HA neurons at the transition from SWS to waking (upper panel) and from waking to drowsy state (lower panel). Each group is represented by a particular symbol, as indicated on the Figure. The pooled mean frequency with 0.1-s bins was obtained from more than 34 transitions observed in 4 HA neurons and 4 or 5 neurons of each POA/BFB neuron group. 0 indicates the onset of EEG activation in the upper panel and the onset of EEG synchronization (deactivation) in the lower panel (see Figs. 5A, 5B, and 9 for details). The statistical significance of the difference between the discharge rate going from SWS to W (upper panel) or from W to drowsy state (lower panel) and the reference discharge rate obtained from the 0.6-s periods of SWS or W, 3 to $2.4 \mathrm{sec}$ prior to the onset of the state transition was examined. The arrows indicate the first point at which a statistically significant decrease in firing rate was observed (MannWhitney). 
Figure 8. Discharge pattern (A) and mean time course of the discharge rate (B) of the three groups of POA/BFB neurons during the transition from SWS to PS. The pooled mean frequency with 0.5 -s bins was obtained from 22 transitions from 22 SWS-specific neurons (squares), 26 from 26 TI-Ss neurons (black dots), and 11 from 10 TIRs neurons (grey dots). 0 indicates the onset of appearance of continuous rhythmic theta waves (see Fig. 5C for details). The statistical significance of the difference between the discharge rate on going from SWS to PS and the reference discharge rate obtained from the 3 -s periods of SWS or W, 10 to $7 \mathrm{sec}$ prior to the onset of the state transition was examined. The arrows indicate the first point at which a statistically significant change in firing rate was observed (Mann-Whitney).

Figure 9. Activity of a representative fast SWS/PS-specific neuron. Note the single spike or cluster discharge during both SWS and PS and complete cessation of discharge during waking. The trace showing the W-D transition (indicated by the bar) is expanded and shown in the box. The dashed lines in the box and the unlabeled arrows indicate the state transitions, while the arrows with an " $\mathrm{s}$ " indicate the arousing sound stimulus.

Figure 10. Activity of a representative PS-specific neuron. Note that activity is highest during PS, the cell being completely silent during W and discharging at a low rate during SWS. The unlabeled arrows indicate the state transitions, while the arrows with a " $\mathrm{t}$ " indicate active waking elicited by touching the tail with a soft brush. The 4 traces in A are from the continuous period indicated by the bar under "a" in B.

Figure 11. Activity of a representative W-specific neuron. Note the single spike or cluster discharge during waking and cessation of discharge during both SWS and PS. The arrows indicate the state transitions.

Figure 12. Mean spontaneous discharge rate of the rapidly $(\geq 9 \mathrm{~Hz})$ or slowly $(<9 \mathrm{~Hz})$ discharging W-specific (A), slowly $(<10 \mathrm{~Hz}$ ) discharging W/PS-active (B), and rapidly $(\geq 10 \mathrm{~Hz})$ discharging W/PS-active (C) neurons across the wake-sleep cycle. Both the slow and fast W/PS-active neurons were subdivided into 2 groups on the basis of their specific (TI-Ss and TI-Rs) or nonspecific (TI-S and TI-R) firing patterns during EEG activation in waking and PS. Note that the difference in discharge rate across the wake-sleep states was highly statistically significant $(P<0.0001$, Kruskal-Wallis) in each group. In $\mathrm{A}$, the difference between the discharge rate in AW and QW was not statistically significant ( $P>0.05$, Mann-Whitney) in either group, nor was the difference between the discharge rate in SWS and PS. In B, the difference between AW and QW was not statistically 
significant $(P>0.05$, Mann-Whitney) in the TI-S group, but was significant $(P<0.05$, Mann-Whitney) in the TI-Ss group. In $\mathrm{C}$, the difference between $\mathrm{AW}$ and $\mathrm{QW}$ was not statistically significant $(P>0.05$, MannWhitney) in either group, nor was the difference between D and SWS. Note also that, in B and C, the difference between AW and PS was not statistically significant ( $P>0.05$, Mann-Whitney). In all other cases, the difference between two successive states was significant $(P<0.05$, Mann-Whitney) in each group.

Figure 13. Activity of simultaneously recorded SWS/PS-specific (SWS/PSs) and W-specific (Ws) neurons across the sleep-waking states. Note the inverse relationship of the unit activity of these two cells. The period of state transitions seen in A (indicated by the bar with "b") is expanded and shown in B. The trace at the transition from SWS to waking in B is further expanded and shown in the box. The arrows in B indicate the state transitions.

Figure 14. Activity of a representative TI-Ss neuron (A) and the spike shape of TI-Ss (B) and TI-Rs (C) neurons. Note the single spike tonic discharge during both waking and PS and cessation of discharge during SWS. Note also that the TI-Ss neuron shows a triphasic broad action potential, whereas the TI-Rs neuron (same as in Fig. 14) shows a biphasic narrow action potential. The arrows indicate the state transitions. D1-D4 indicate the duration of the averaged action potential measured from onset (t0) to the positive peak (D1), the first zero crossing (D2), the negative peak (D3), and the second zero crossing (D4).

Figure 15. Activity of a representative TI-Rs neuron. Note the single spike or cluster discharge during W and the high frequency burst firing during PS. The arrow indicates the transition from $\mathrm{W}$ to $\mathrm{D}$, while the arrowhead indicates the transition from SWS to W.

Figure 16. Activity and analysis of a representative TI-Rs neuron during PS. Note the rhythmic bursting activity in close relation to theta waves (upper trace), as seen in the peri-theta wave spike histogram (lower trace).

Figure 17. Antidromic responses of a TI-Ss neuron following stimulation of the medial frontal cortex. A, Antidromic action potentials and a collision (shown by arrowhead) with a spontaneous action potential. Four superimposed sweeps. B, Antidromic action potentials $(1,2)$ and collisions with a spontaneous action potential $(3,4)$. Note the fixed latency of the antidromic responses in B1 (five superimposed sweeps). 
Figure 18. Orthodromic responses of an SWS-active (A), a W-active (B), and an SWS/PS-specific (C) neuron following stimulation of the medial frontal cortex.

Figure 19. Diagram illustrating the forebrain mechanisms underlying the alteration of sleep and waking. The SWS-specific (SWS-on) and SWS/PS-specific (SWS/PS-on) neurons in the POA and BFB are proposed to be GABAergic, while the W/PS-specific (W/PS-on) neurons, in particular the TI-Ss neurons, are thought to be cholinergic and the W-specific (W-on) neurons in the POA and BFB glutamatergic. The SWS-on and SWS/PSon neurons function as sleep-promoting neurons, while all W-on and W/PS-on neurons in the forebrain (POA, $\mathrm{BFB}$ and $\mathrm{PH}$ ) and brainstem function as waking-promoting neurons. Initiation of sleep is caused by decreased activity (disfacilitation) of the waking-promoting neurons, which disinhibit the sleep-promoting neurons, thereby enhancing the sleep process. 